\title{
Migração proativa de máquinas virtuais para aplicações móveis na computação em névoa
}

\author{
Diogo M. Gonçalves ${ }^{1}$, Luiz F. Bittencourt ${ }^{1}$, Edmundo M. R. Madeira ${ }^{1}$ \\ ${ }^{1}$ Instituto de Computação - Universidade Estadual de Campinas (UNICAMP) \\ Campinas - São Paulo - Brasil \\ diogomgelrc.ic.unicamp.br, \{bit, edmundo\}@ic.unicamp.br
}

\begin{abstract}
Applications often utilize the cloud as support for processing and storage. The variety of mobile applications also brings a diversity of quality of service requirements, as for example strict delay and availability requirements. Fog computing includes computing services at the edge of the network so as response times can be reduced. In this paper, we present one policy for proactive virtual machine migration in fog computing in order to improve management of computing resources utilized by vehicles connected to this infrastructure. Simulations suggest that using knowledge about the future user's path can improve the resource management of fog ecosystem, maintaining users' virtual machine in fog devices as close as possible to the vehicle path. Simulations suggest that the presented policy reduce the total of migration along the user's path without affecting the quality response time of virtual machines allocated to the fog.
\end{abstract}

Resumo. Aplicações frequentemente utilizam nuvens como suporte ao processamento e armazenamento. A diversidade de aplicações móveis traz também uma diversidade de requisitos de qualidade de serviço, tais como requisitos estritos de atraso e disponibilidade de acesso. A computação em névoa inclui recursos computacionais na borda da rede para que o atraso no tempo de resposta possa ser reduzido. Neste artigo, apresentamos uma política de migração proativa de máquinas virtuais em ambientes de névoa a fim de melhorar o gerenciamento dos recursos computacionais utilizados pelos veículos conectados a essa infraestrutura. Simulações sugerem que a utilização de predições sobre o trajeto futuro do veículo pode melhorar o gerenciamento de recursos da névoa, mantendo a máquina virtual do usuário em dispositivos de névoa tão próximos quanto possível do trajeto do automóvel. A solução apresentada reduz o número de migrações realizadas durante o trajeto do usuário sem prejudicar o tempo de resposta da máquina virtual alocada na névoa.

\section{Introdução}

A incorporação de sensores e tecnologias de comunicação aos veículos e, consequentemente, a criação de redes veiculares (Vehicular Ad Hoc Network - VANET), têm contribuído para o desenvolvimento de aplicativos inteligentes relacionados ao contexto do veículo, auxiliando em decisões de segurança e trajeto, além de aumentar o conforto dos usuários [Yan et al. 2013].

A crescente gama de aplicativos móveis que podem ser incorporados neste contexto, tanto diretamente no veículo quanto pelos seus passageiros através de seus smartphones, por exemplo, resulta em um aumento da demanda por recursos de processamento e armazenamento nesse ambiente. Os veículos, no entanto, geralmente não oferecem recursos computacionais suficientes para processamento e armazenamento de 
todas as aplicações. Em um ambiente de transporte coletivo, por exemplo, um grande grupo de usuários possuem variadas demandas por recursos a partir das aplicações de seus interesses. Uma alternativa proposta para este problema foi possibilitar o acesso a recursos remotamente disponíveis. Estudos têm mostrado que a migração do processamento de aplicativos de dispositivos com pouco poder computacional para servidores remotos permite uma diminuição no tempo de processamento dessas aplicações [Nabi et al. 2015, Júnior et al. 2016].

Aplicativos mais robustos podem se apoiar em conceitos da Computação em Nuvem (Cloud Computing) ao utilizarem fontes mais ricas em recursos para executar suas requisições. As características e/ou a qualidade da conexão com esses servidores, porém, pode comprometer o funcionamento de aplicações que necessitam de uma baixa latência na comunicação. Para amenizar esse cenário, conceitos de Computação em Névoa (Fog Computing) têm sido incorporados no contexto de redes veiculares: servidores menores, denominados Roadside Cloudlets, podem ser posicionados ao lado das vias para oferecer um conjunto de recursos mais próximos aos usuários, diminuindo atrasos de comunicação. Esses servidores são acessíveis somente pelos veículos dentro do raio de cobertura das Roadside Units, interfaces via rádio que permitem a conexão entre o usuário e os recursos dos servidores.

$\mathrm{O}$ acesso a esses recursos encontra um problema intrínseco aos veículos que fazem uso desse serviço: seu deslocamento. Devido à mobilidade dos veículos, o conteúdo localizado remotamente tende a afastar-se do veículo, quando preferencialmente deveria estar próximo ao usuário para ser acessado no menor período de tempo possível. Aplicações interativas ou em tempo-real, por exemplo relacionadas a jogos e serviços de multimídia, necessitam de uma baixa latência para proporcionarem uma boa experiência ao usuário.

Usuários de computação em nuvem frequentemente possuem máquinas virtuais (VMs) com aplicações e dados localizadas fixamente em um centro de dados do provedor de nuvem. No contexto de aplicações móveis na computação em névoa, posicionar as máquinas virtuais de forma que sua localização física beneficie o usuário é uma das chaves para garantir uma boa execução dos aplicativos remotamente [Kim et al. 2015].

Devido à grande mobilidade característica de alguns dispositivos da Internet das Coisas, como os veículos, a definição de mecanismos para tomada de decisão sobre o momento e o destino ótimos para o processo de migração em servidores na névoa se torna não trivial, ainda sendo um problema de pesquisa em aberto [Puliafito et al. 2017, Bittencourt et al. 2015, Roman et al. 2016].

Desenvolver bons mecanismos para o gerenciamento de máquinas virtuais em um ambiente de computação na névoa não só tem sido apontado como uma das chaves para possibilitar um "aumento substancial de soluções com suporte à mobilidade, tanto em termos de performance quanto de aplicabilidade"[Puliafito et al. 2017], como também está entre os desafios para o desenvolvimento da Internet do Futuro para Cidades Inteligentes [Batista et al. 2016].

Algumas soluções têm sido propostas para manter a máquina virtual no servidor melhor localizado para o usuário a fim de diminuir a latência na comunicação [Yao et al. 2015, Refaat et al. 2014, Yu et al. 2013b]. No entanto, esses trabalhos visam apenas corrigir o posicionamento da máquina virtual após o deslocamento do usuário, atuando de forma reativa. Este trabalho apresenta uma abordagem proativa quanto ao processo de migração de máquinas virtuais em um ambiente de névoa. Tendo como base dados de mobilidadede uma rede de transporte coletivo, bem como informações sobre a 
localização futura dos veículos, a política de migração proposta visa antecipar a migração do conteúdo do usuário para uma Cloudlet que provavelmente fará parte do trajeto do usuário no futuro próximo.

Simulações apontam que a abordagem proposta neste artigo permite um aumento na disponibilidade do sistema a partir da minimização no número de migrações necessárias durante o trajeto do usuário. Tais benefícios são obtidos sem comprometer outros parâmetros de avaliação, como latência e volume de dados trafegados.

O restante deste artigo está organizado da seguinte forma. A Seção 2 descreve conceitos básicos e definições utilizadas neste trabalho, como computação em névoa, virtualização e redes veiculares. A Seção 3 apresenta o estado da arte relativo à pesquisa sobre migração de máquinas virtuais em névoa e utilização de predição de mobilidade para otimizar o gerenciamento de recursos. A Seção 4 discorre sobre a abordagem proativa proposta neste trabalho. A Seção 5 descreve a metodologia adotada no processo de implementação e avaliação da proposta. Por fim, a Seção 6 apresenta os resultados obtidos por meio de simulações seguindo os critérios descritos na Seção 5.

\section{Conceitos Básicos}

Esta seção apresenta os conceitos básicos relacionados aos assuntos contidos neste artigo, como computação em nuvem e névoa, Vehicular Cloud, virtualização e migração de máquinas virtuais.

\subsection{Computação em nuvem}

A demanda pela otimização do uso de recursos computacionais, a melhoria na qualidade dos transmissão e o barateamento de equipamentos e processamento influenciaram o desenvolvimento de um novo modelo de computação, no qual o acesso a esses recursos é feito remotamente e sob demanda.

O modelo de Computação em Nuvem (Cloud Computing) [Mell and Grance 2011] oferece o acesso a um conjunto de recursos, tais como armazenamento, processamento, aplicativos e serviços de forma que a requisição e liberação desses recursos seja feita de forma rápida e transparente ao usuário.

$\mathrm{O}$ acesso ao hardware, geralmente distribuído, oferecido pela Cloud é feito principalmente por meio de virtualização. A virtualização é uma tecnologia que abstrai os recursos físicos do hardware e oferece o acesso à eles por meio de máquinas virtuais (do inglês, Virtual Machine - VM). Este processo de migração do processamento e armazenamento para servidores na nuvem permitiu que aplicações mais robustas pudessem ser executadas em dispositivos móveis seguindo este mesmo princípio [Ahmed et al. 2015], dando origem a Computação Móvel nas Nuvens (Mobile Cloud Computing - MCC).

Algumas limitações relacionadas à arquitetura da Cloud Computing tais como latência elevada, atraso e perda de pacotes levaram à aproximação de alguns servidores para as bordas da rede como tentativa de amenizar os danos causados a aplicações de tempo real. Os autores em [Satyanarayanan et al. 2009] apresentam este conceito de Cloudlet, servidores próximos aos usuários acessados através de tecnologias sem fio em no máximo um salto na rede. O acesso aos recursos da Cloudlet, assim como na Cloud, podem ser feitos através de máquinas virtuais.

\subsection{Computação em névoa}

A popularização de dispositivos que incorporam o acesso à Internet para melhorar sua experiência com o usuário, como eletrodomésticos, veículos e máquinas industriais, clas- 
sificados como parte da Internet das Coisas (Internet of Things - IoT), trouxeram novas demandas relacionadas à computação em nuvem.

O baixo poder de processamento e armazenamento desses dispositivos levou a uma demanda por uma fonte de recursos mais abundante, como a nuvem, para gerenciar um volume mais crítico de dados. A demanda por mobilidade e baixa latência por parte dos dispositivos IoT, no entanto, tornou a comunicação com servidores localizados muito longe desses dispositivos um ponto crítico relacionado ao desempenho dessas aplicações.

Com base nesse cenário, [Bonomi et al. 2012] propuseram o conceito de Fog Computing, "uma plataforma altamente virtualizada que provê processamento, armazenamento e serviços de rede entre os dispositivos e os tradicionais Data Centers em nuvem, tipicamente mas não exclusivamente localizada na borda da rede”. A Figura 1 ilustra uma arquitetura de típica de um ambiente de névoa computacional, bem como a indicação de possíveis fluxos de migração de dados de usuários móveis [Bittencourt et al. 2015].

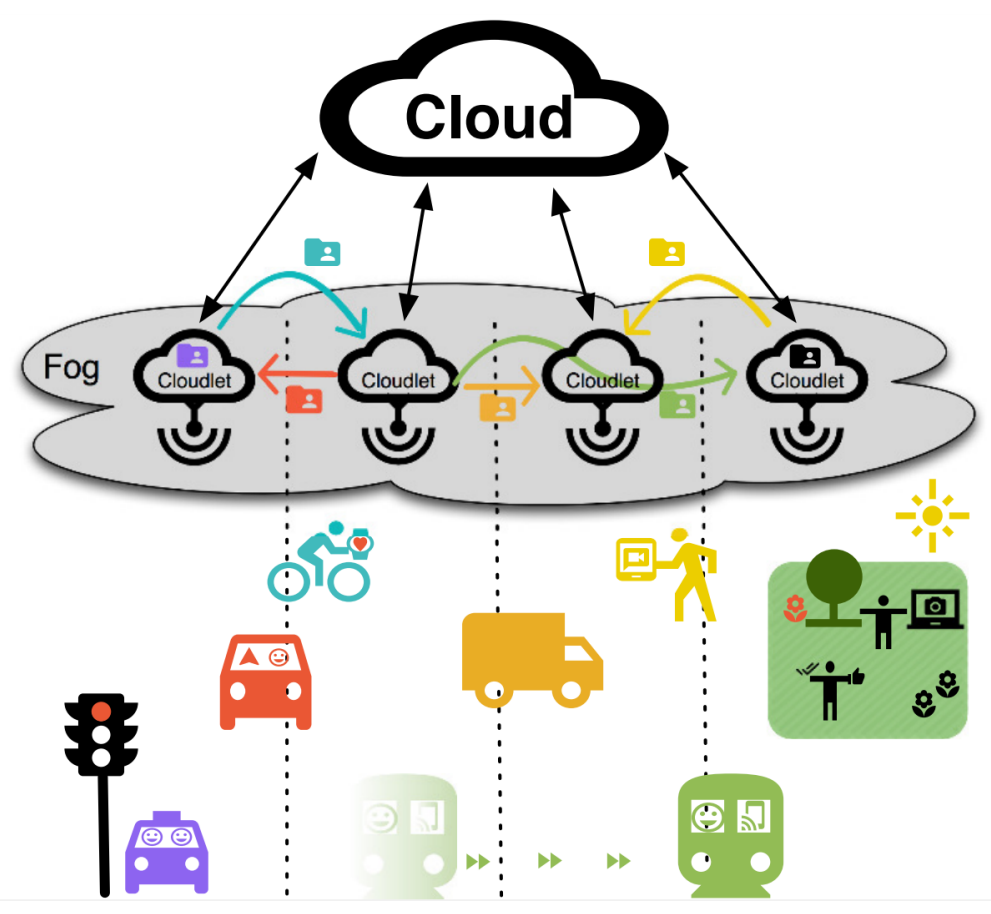

Figura 1. Arquitetura de um ambiente de computação em névoa para atender usuários móveis [Bittencourt et al. 2015]

\subsection{Redes veiculares e a integração com a névoa}

As redes veiculares (Vehicular Ad-hoc Networks - VANET) são classificadas como um conjunto de carros conectados entre si por meio de tecnologias sem fio. As VANETs são compostas por conexões veículo-a-veículo (V2V) e veículo-a-infraestrutura (V2I).

A conectividade entre os veículos oferecida pelas VANETs permitiu o acesso e compartilhamento de recursos externos aos veículos. A Vehicular Cloud Computing (VCC) é formada por um grupo de veículos que compartilham seus recursos de processamento e armazenamento com os demais veículos da rede.

A incorporação de conceitos de computação em névoa para este contexto permitiu uma clara arquitetura escalável para esse cenário. [Yu et al. 2013a] descrevem uma ar- 
quitetura hierárquica para névoa em redes veiculares composta por 3 camadas: Vehicular Cloud, Roadside Cloudlet e Central Cloud.

Uma Vehicular Cloud é formada por um grupo de veículos que compartilham seus recursos entre si; A Roadside Cloudlet é formada por Cloudlets locais acessadas através das Roadside Units (RSU), interfaces via rádio responsáveis pela comunicação com os veículos. A Roadside Cloudlet é acessível somente pelos veículos dentro do raio de cobertura das RSUs. Quando o veículo passa a ficar sob domínio de outra RSU, iniciase a dependência de nós intermediários para conectar o usuário à sua máquina. Este problema pode ser amenizado realizando a transferência da maquina virtual da Cloudlet antiga para a atualmente associada à RSU na qual o usuário está conectado. Por fim, uma Central Cloud é composta por servidores de maior capacidade localizados em centros de dados e acessados quando há uma grande demanda por recursos.

$\mathrm{O}$ acesso a esses recursos externos geralmente é realizado por meio de máquinas virtuais. A migração de máquinas virtuais consiste na transferência da localização física da máquina virtual entre os servidores da rede, seja para realizar um balanceamento de carga, manutenção física no servidor ou ajustar a localização da máquina de acordo com a posição do usuário. A live-migration permite que VM seja transferida sem que, durante o processo, o usuário tenha indisponibilidade significativa nos recursos utilizados.

Nesse sentido, o conceito de Follow-me Cloud apresentado em [Taleb and Ksentini 2013] propõe que, dada uma rede com um conjunto de pequenos servidores geograficamente distribuídos em sua borda, uma vez que o usuário se movimente dentro da rede, seu conteúdo presente nos servidores deve segui-lo, mantendo a qualidade e a disponibilidade do acesso a ele.

\section{Trabalhos Relacionados}

A maioria das soluções propostas para o problema de migração de conteúdo apenas reagem ao deslocamento do usuário, iniciando o processo apenas quando alguma métrica utilizada, como número de saltos ou latência, atinge um limiar especificado. Alguns trabalhos no contexto de Mobile Cloud e Vehicular Cloud apresentaram bons resultados ao incorporar uma predição de mobilidade do usuário a fim de migrar proativamente o conteúdo de interesse do usuário para regiões que farão parte de seu trajeto em um futuro próximo. No contexto de computação em névoa, as atuais abordagens ainda não utilizam essas informações sobre o trajeto do usuário para melhorar o processo de migração. $\mathrm{O}$ estado da arte relativo ao processo de migração de máquinas virtuais em um ambiente de névoa e trabalhos que utilizam informações do futuro trajeto do usuário para otimizar a transferência de dados mas que, até o momento, só foram aplicados em outros contextos, são descritos com mais detalhes abaixo.

Os autores em [Yao et al. 2015] apresentam um estudo focado no processo para determinar o momento e o destino adequado da migração a fim de minimizar os custos de rede. Assim como a abordagem proposta neste estudo, o trabalho citado tem como base a transferência de VMs entre Vehicular Cloudlets e realiza o cálculo de otimização de forma individual e a partir da visão do cliente, ou seja, o processo de decisão é feito a fim de minimizar os custos relacionados a cada cliente e não considerando ações que beneficiariam um grupo de usuários. Apesar disto, o processo de migração é feito de forma reativa, ou seja, com base no deslocamento que o veículo já realizou, não sendo tomadas decisões com base em alguma previsão de deslocamento.

Apesar de considerar o deslocamento dos veículos, a abordagem apresentada em [Refaat et al. 2014] prioriza a migração das máquinas virtuais entre os veículos, não se be- 
neficiando de recursos externos que as Roadside Cloudlets oferecem. Os veículos nesse contexto são utilizados como fonte de recursos para atender a uma demanda de processamento da rede. O acesso a infraestruturas fixas se limita a utilização de Roadside Units como intermediárias no processo de migração entre veículos distantes. A política de migração também se limita a uma abordagem reativa quanto ao deslocamento do veículo.

Os trabalhos [Gomes et al. 2016b, Gomes et al. 2016a] avaliam a migração das máquinas virtuais considerando possíveis destinos dos usuários de rede LTE, porém o foco desses trabalhos está na seleção de dados para caching. Com isso o processo de migração visa otimizar a localização do conteúdo a partir de uma visão global dos dados, tomando decisões que beneficiem um grupo que estará acessando o conteúdo.

Os autores em [Gomes et al. 2016b] propõem um modelo baseado em Mobile Follow-me Cloud para migração de máquinas virtuais. Devido à mobilidade dos usuários, o estudo utiliza uma abordagem para a migração de VM de acordo com os conteúdos populares à certos grupos de usuários. Diferentemente da abordagem proposta neste trabalho, o processo de decisão apresentado por Gomes et al. baseia-se na otimização global da rede, o que pode não oferecer e a configuração mais adequada para cada usuário.

O trabalho [Gomes et al. 2016a] apresenta uma estratégia para migração de máquinas virtuais baseada na previsão de deslocamento dos usuários. A abordagem considera probabilidades de destinos para cada usuário individualmente além de dados relacionados aos destinos da VM como espaço disponível, número de usuários conectados e o custo da migração. Apesar de cada usuário calcular os possíveis destinos para o conteúdo que ele está acessando, o servidor responsável pelo processo de migração decide pela movimentação dos dados apenas quando um grupo de usuários requisita a migração.

Os autores apresentam em [Malandrino et al. 2014] um modelo para a definição da fontes do conteúdo de interesse do usuários baseado nos recursos disponíveis na sua localização. Com base nos dados de mobilidade e conteúdo consumido pelos usuários, as Roadside Units selecionam as melhores fontes para o download de conteúdo de interesse dos usuários e quais partes do conteúdo estarão disponibilizadas nessa fonte. A partir de uma predição da mobilidade do usuário, a continuidade do conteúdo utilizado por ele pode ser preparado em fontes próximas que deverão ser utilizadas no futuro. O modelo assume Roadside Units e veículos como fontes de conteúdo e, em casos em que essas fontes não estão disponíveis, a conexão é feita diretamente com servidores da nuvem através de conexões celulares. Apesar de utilizar uma predição de mobilidade para otimizar o deslocamento dos dados dos usuários, o trabalho não utiliza os recursos que a névoa pode oferecer para otimizar o processamento de aplicações de interesse dos usuários.

O trabalho proposto pelos autores em [Shin et al. 2015] apresenta uma abordagem para utilização de potenciais caches móveis, como veículos, para transportar dados solicitados por usuários de dispositivos móveis, aumentando a taxa de transferência da rede e diminuindo o uso de recursos da rede celular. Após a solicitação de um dado da nuvem, o sistema transfere os dados para bases fixas na via e em seguida para um veículo que possui uma rota que passará pela localidade do usuário, permitindo que o usuário receba seus dados através de uma fonte mais próxima. O trabalho apresenta o potencial de se utilizar informações sobre o trajeto dos usuários para otimizar a transferência de dados de interesse dos usuários, mas não utiliza essa abordagem no contexto de aplicações que também demandam um poder de processamento.

O trabalho apresentado em [Mustafa et al. 2017] propõe a utilização de uma predição de mobilidade do trajeto do usuário para otimizar o processo de migração de 
máquinas virtuais em Vehicular Cloud. Apesar de também explorar informações sobre o destino do usuário, a principal fonte de recursos para realizar o processamento requisitado pelo usuário são os próprios veículos que compõem a rede. No escopo do trabalho, as Roadside Units são utilizadas apenas como intermediárias no processo de migração das máquinas entre os veículos, não fazendo uso efetivo dos recursos disponíveis na névoa. $\mathrm{O}$ trabalho sugere que a utilização de informações do futuro trajeto do veículo melhoram a qualidade de serviço oferecida ao usuário, apesar de utilizar outras métricas para realizar essa afirmação, como número de tarefas requisitadas pelo usuário executadas com sucesso e não utilizando outros critérios de avaliação essenciais no contexto de computação em nuvem, como latência e disponibilidade de acesso.

Como visto nos trabalhos citados acima, incorporar dados acerca da localização futura dos usuários podem trazer um aumento na qualidade de serviço oferecido aos usuários móveis. Esta abordagem porém, ainda não foi explorada para o benefício de aplicações que também requerem maior poder de processamento e baixa latência. Esta demanda por processamento e, ao mesmo tempo, latência reduzida pode ser suprida pelos recursos da névoa. As atuais propostas para melhorar o processo de migração de máquinas virtuais em ambientes de névoa não se aproveitam dessas informações do trajeto do usuário. O objetivo deste trabalho é propor e avaliar uma abordagem para utilização de computação em névoa como suporte a aplicações em veículos de forma que latência seja minimizada através da migração de máquinas virtuais na borda da rede.

\section{Abordagem proposta}

A partir do cenário descrito acerca das atuais políticas de migração de máquinas virtuais em ambientes de computação em névoa, e dos benefícios que sua aplicação em outros contextos obtiveram ao incorporar informações sobre o trajeto futuro de seus usuários às suas políticas de gerenciamento de recursos, este trabalho propõe a combinação destes dois conceitos, incorporando predição de mobilidade aos critérios utilizados para a escolha do local de destino de um pedido de migração de máquina virtual.

Políticas de migração de VMs podem escolher o destino das máquinas virtuais com base na menor distância física ou latência imediata apresentada entre o usuário e a Cloudlet. Apesar desse tipo de política geralmente oferecer a menor latência possível para o usuário em um primeiro momento, após a migração em pouco tempo o usuário passará a se distanciar novamente do seu conteúdo, antecipando a necessidade de se realizar uma nova migração. Neste trabalho propomos realizar a migração para um local à frente do veículo. Dessa forma, o usuário passará a se aproximar do seu conteúdo em um primeiro momento e só após algum tempo que ele passará a se distanciar novamente, até surgir se necessite repetir o processo de migração.

A partir do momento em que o sistema detectar a necessidade de se realizar uma nova migração para corrigir a localização da VM com relação à atual posição do veículo, a política proposta irá selecionar as Cloudlets que estão disponíveis na área de interesse do veículo. Com o conjunto de Cloudlets selecionadas, o destino da máquina virtual será escolhido de acordo com a menor latência apresentada para aquela área, respeitando os recursos disponíveis pelas Cloudlets.

A Figura 2 ilustra um exemplo de migração de máquina virtual ao longo do caminho de um usuário utilizando a política proposta. Ao identificar a necessidade de se realizar uma migração, inicia-se o processo para calcular a localização futura do usuário (indicada pela linha tracejada) com base no período de predição que está sendo utilizado. A partir da localização futura, uma área de interesse do usuário é criada e todas as Clou- 
dlets presentes nesta área se tornam candidatas a receber a máquina virtual do usuário, priorizando a melhor latência e respeitando os recursos disponíveis.

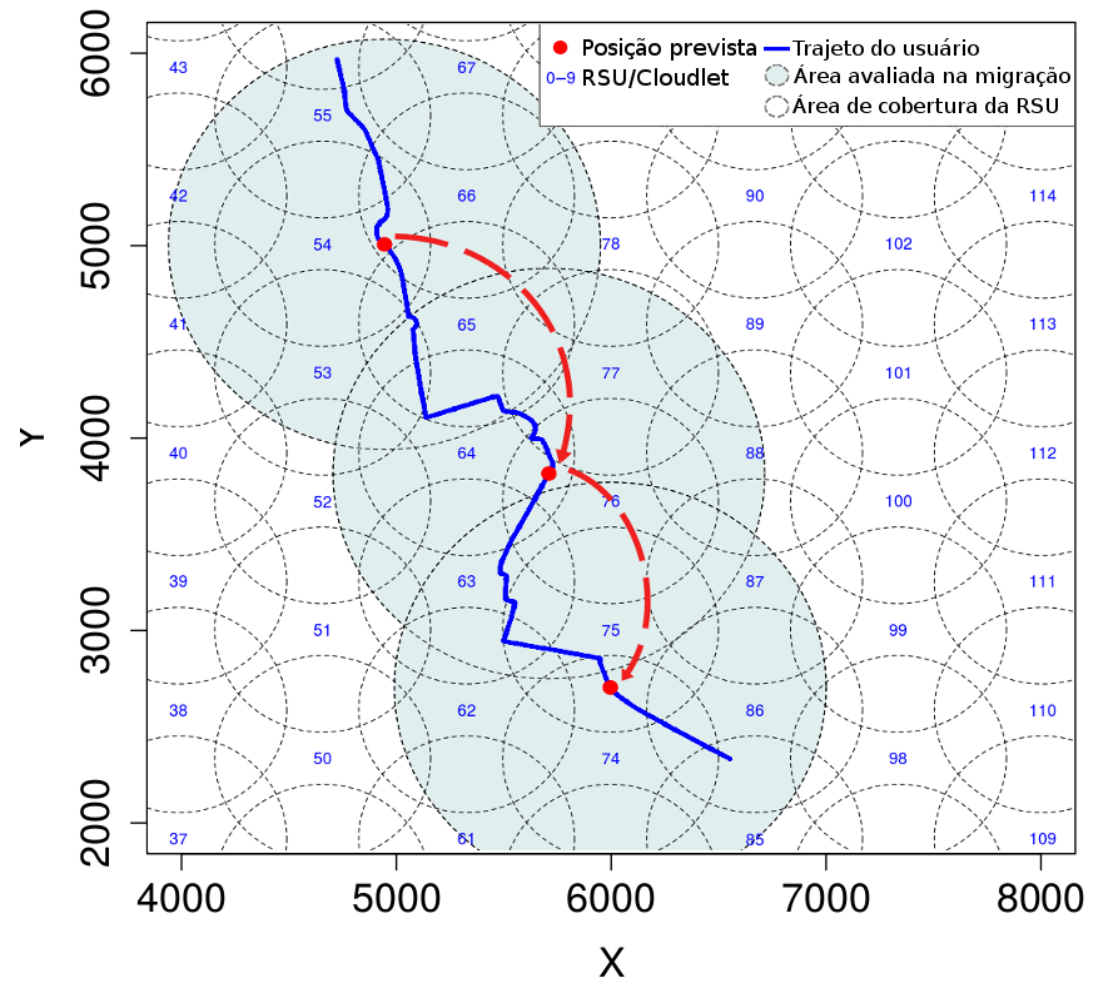

Figura 2. llustração do processo de migração entre os nós da Fog.

O destino do conteúdo do usuário deve ser escolhido ,preferencialmente, tão à frente no caminho do usuário quanto possível, desde que a distância entre o usuário e a Cloudlet não comprometa os níveis de latência exigidos pelas aplicações. A minimização no número de migrações está diretamente relacionada a maximização do periodo de tempo em que uma Cloudlet consegue atender as necessidades do usuário.

O tempo em que o usuário manter-se-á conectado à Cloudlet pode ser otimizado escolhendo o servidor melhor localizado de acordo com seu trajeto nos próximos minutos. Quanto maior o conhecimento sobre o trajeto futuro do veículo, melhores são os dados disponíveis para escolher o destino para aplicação. O intervalo de tempo futuro em que se tem conhecimento da localização do usuário é referenciado neste artigo como tempo de predição. Foram realizadas simulações em variados cenários para identificar um comportamento do processo de escolha do destino das máquinas virtuais de acordo com o tempo de predição disponível.

A fim de avaliar se a abordagem proposta poderia obter resultados promissores para o processo de migração, optou-se por uma avaliação mais otimista quanto a acurácia da previsão de localização futura dos usuários. Esta escolha evita que a abordagem seja comprometida com a acurácia dos algoritmos de predição encontrados na literatura. Resultados ruins nesta fase de avaliação poderiam indicar uma alta probabilidade de não se obter benefícios ao incluir esta métrica em outras propostas de migração, visto que a utilização de algoritmos reais de predição fatalmente apresentariam uma menor acurácia com relação a localização prevista para o usuário. Tal taxa poderia mitigar os possíveis ganhos obtidos pela abordagem. 
Com base nesse contexto, escolheu-se um cenário em que pode-se obter uma alta acurácia em relação à predição de localização dos usuários. Neste estudo foram consideradas rotas de ônibus urbanos como base para a mobilidade dos usuários. Devido ao seu trajeto fixo e um deslocamento relativamente previsível, as rotas dos ônibus apresentam um bom cenário para iniciar a avaliação desta abordagem.

\section{Metodologia}

Para a avaliação da abordagem proposta foi utilizado como base o simulador MyIFogSim [Lopes et al. 2017], uma extensão do simulador IFogSim [Gupta et al. 2017] que permite a inclusão de usuários móveis conectados à nevoa. A fim de avaliar o processo de migração a partir de um veículo, o modelo de mobilidade utilizado para esses usuários foi baseado no percurso de 500 ônibus da cidade de Luxemburgo [Codeca et al. 2015]. Para a utilização dos percursos dos ônibus foi necessário realizar uma integração entre o simulador MyIFogSim e o simulador SUMO (Simulator for UrbanMObility) ${ }^{1}$, responsável pela leitura e interpretação da base de dados. A partir dos dados de localização fornecidos pelo SUMO, uma nova base da dados foi gerada de forma otimizada para o contexto deste trabalho. Esta nova base de dados foi utilizada posteriormente como base para definir a mobilidade dos usuários no simulador MyIFogSim.

O simulador MyIFogSim permite uma avaliação do comportamento de aplicações relacionadas a dispositivos característicos da Internet das Coisas e a computação na névoa. Dentre os recursos oferecidos pelo simulador estão o gerenciamento do processo de handoff entre as estações base, além ferramentas para a realização e avaliação dos processos de offloading e migração de máquinas virtuais. O simulador oferece alguns modelos para a migração das máquinas virtuais. O modelo utilizado neste trabalho é o processo de migração ao vivo (live migration), que consiste na transferência de partes da máquina virtual da máquina física de origem para o local de destino enquanto a aplicação ainda está em execução. Essa técnica diminui o tempo em que usuário fica impossibilitado de acessar sua aplicação durante o processo de migração.

Cada Cloudlet responsável pelo processamento das aplicações foi configurada com um poder de processamento de 2,8 MIPS (Milhões de instruções por segundo), 25 Gigabytes de memória RAM e largura de banda de 1 Gigabit por segundo; cada Cloudlet foi conectada a um ponto de acesso através de um link de 100 Megabits por segundo e uma latência de 4 milissegundos. O raio de cobertura de cada ponto de acesso é de 500 metros.

A proposta foi avaliada analisando o comportamento das migrações a partir do conhecimento do trajeto futuro do veículo. Tais dados foram obtidos diretamente do trajeto recebido como entrada na simulação. Para cada um dos 500 trajetos de ônibus avaliados foram realizadas 6 simulações, cada uma tendo conhecimento do trajeto futuro do veículo dentro de um intervalo de tempo pré-definido, onde tais valores variaram de 0 a 300 segundos. O tempo de predição 0 não considera quaisquer informações sobre as futuras posições do veículo, sendo utilizada como base para comparação com a abordagem proposta. As métricas adotadas para a avaliação foram latência, número de absoluto de migrações, tempo de utilização da máquina virtual sem que se necessite realizar uma migração, tempo de indisponibilidade de acesso à máquina durante a migração, volume de tráfego de dados gerado e tempo de migração.

Para avaliar se características intrínsecas ao domínio dos usuários móveis avaliados neste trabalho, como velocidade e duração do trajeto, apresentavam algum impacto

\footnotetext{
${ }^{1}$ http://sumo.dlr.de
} 
de desempenho no processo de migração, os veículos foram agrupados em 3 conjuntos de acordo com sua velocidade média durante o trajeto. Dados acerca do conjunto de entrada utilizado podem ser vistos na Tabela 1.

Tabela 1. Característica do dataset

\begin{tabular}{lll}
\hline \hline Conjunto & Duração média do Percurso (min) & Quantidade \\
\hline$<20 \mathrm{~km} / \mathrm{h}$ & 25,54 & 192 \\
$>=20 \mathrm{~km} / \mathrm{h} \&<25 \mathrm{~km} / \mathrm{h}$ & 26,44 & 223 \\
$>=25 \mathrm{~km} / \mathrm{h}$ & 9,45 & 85 \\
Média geral & 23,31 & 500 \\
\hline \hline
\end{tabular}

Para cada combinação de métrica e conjunto avaliado, os dados são apresentados na forma de média entre todos os indivíduos do conjunto e ilustrados dentro de um intervalo de confiança de $95 \%$.

\section{Resultados}

A Figura 3(a) apresenta o número total de migrações realizadas durante todo o trajeto do veículo. Nota-se uma tendência na redução do número de migrações realizadas com o aumento no tempo de predição. Em média, os grupos chegaram a apresentar uma redução de até $20 \%$ no número total de migrações ao utilizar um conhecimento sobre o trajeto dos próximos 5 minutos do veículo.

A disparidade no número de migrações relativa ao grupo de veículos rápidos em comparação aos demais pode ser explicada a partir das características do seu trajeto. $\mathrm{O}$ número absoluto de migrações está relacionado a distância percorrida por cada veículo. Assumindo intuitivamente a correlação entre a distância percorrida e o tempo despendido realizando tal trajeto, pode-se considerar que o gráfico apresenta uma escala coerente entre os grupos avaliados, tendo em vista as características apresentadas na Tabela 1.

Apesar da redução no número absoluto de migrações ser um indicativo de que houve uma diminuição na necessidade de realizar uma migração, essas diferenças de trajeto apresentadas pelos grupos de veículos rápidos acabou diminuindo a percepção dos ganhos relativos obtidos por cada grupo. Para complementar a figura anterior, a Figura 3(b) apresenta o tempo médio em que as máquinas virtuais ficam hospedas em cada Cloudlet antes que uma nova migração fosse necessária. O comportamento padrão apresentado pelos grupos foi de um crescimento no tempo de hospedagem da máquina a medida que se aumentava o conhecimento sobre o trajeto do veículo. Percebe-se que a velocidade dos veículos interfere no tempo de hospedagem da máquina virtual em cada máquina física. A medida em que a velocidade aumenta, menor o tempo necessário para que uma nova migração necessite ocorrer.

A Figura 4(a) apresenta o tempo necessário para realizar o processo de migração entre as Cloudlets. Percebe-se que todos os grupos apresentam uma pequena tendência a diminuir o tempo de migração à medida que se aumenta o tempo de predição realizado. A partir de um processo de migração mais rápido, o tempo de indisponibilidade de acesso à máquina virtual, a cada migração, tende a diminuir. A Figura 4(b) ilustra esse comportamento.

Apesar da diminuição do número de migrações poder sugerir que os locais no qual as máquinas virtuais estão sendo posicionadas estão sendo mais adequadas ao usuário, uma métrica importante a se avaliar no contexto de aplicações relacionadas a computação 


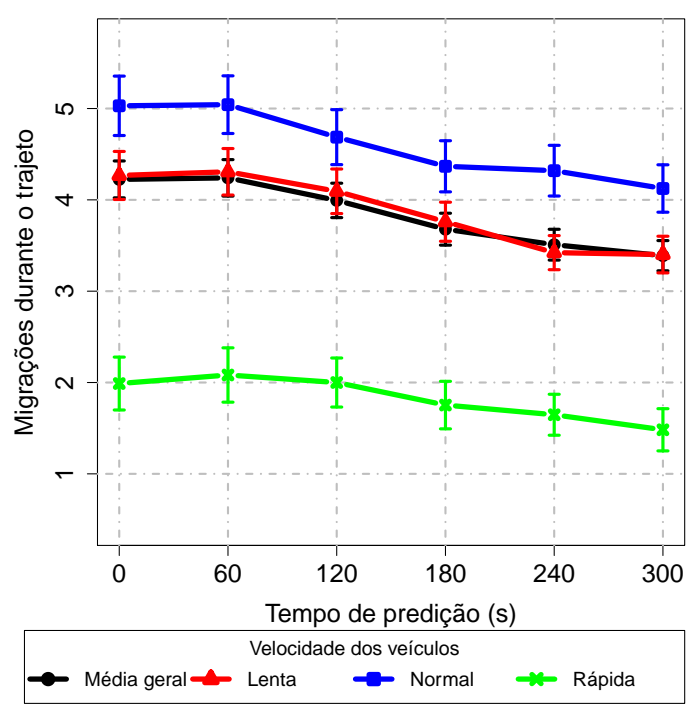

(a) Número de migrações durante o trajeto

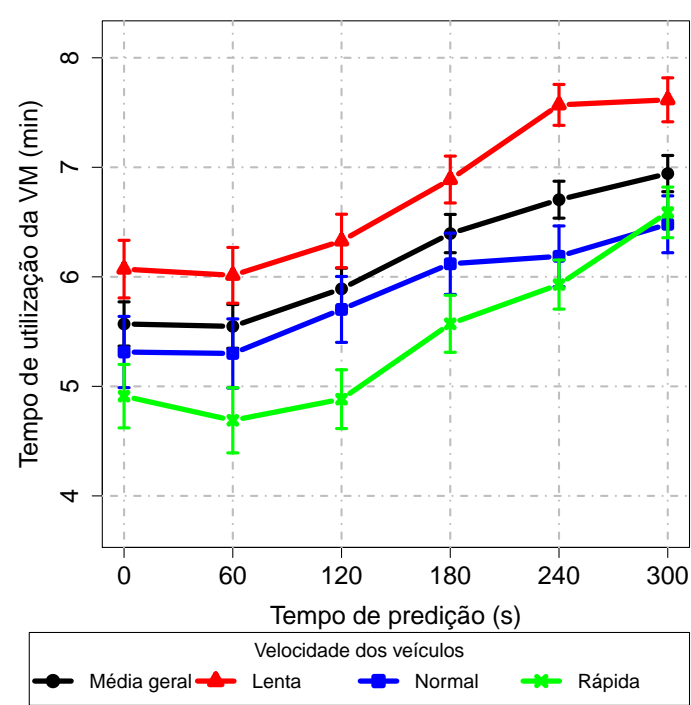

(b) Intervalo de tempo entre migrações

Figura 3. Relação número de migrações e período de disponibilidade da máquina para o usuário.

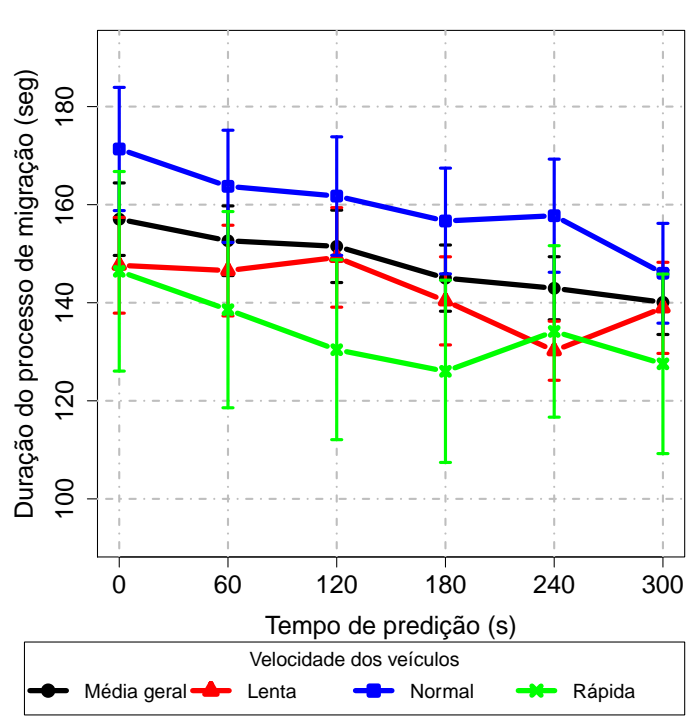

(a) Tempo de duração do processo de migração da máquina virtual entre as Cloudlets.

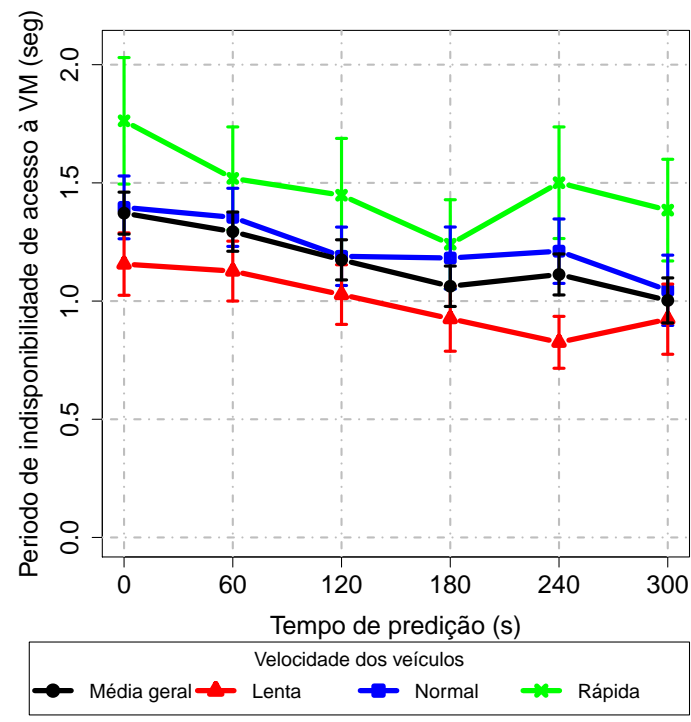

(b) Tempo médio em que o veículo ficou sem acesso à máquina virtual a cada migração.

Figura 4. Tempo necessário para a realização de uma migração e o tempo de indisponibilidade de acesso à máquina durante esse processo.

em névoa é a latência. Um aumento na distância entre o usuário e sua aplicação pode comprometer os níveis de latência exigidos em seu contexto. Considerando o intervalo de predição avaliado, as simulações realizadas não indicaram um aumento significativo da latência a partir da média geral e do pior caso encontrado. Mesmo com a redução no número de migrações, a latência não foi comprometida dentro do intervalo avaliado. Pode-se ver na Figura 5(a) que a latência média se mostrou estável em todos os casos e o pior não apresentou aumento estatisticamente significativo, com sobreposição dos intervalos de confiança dos pontos da curva. 
As reduções no número de migrações e no tempo utilizado nesse processo contribuem para um aumento no tempo de disponibilidade da máquina virtual para o usuário, pois, durante parte do processo de migração, o usuário fica impossibilitado de acessar a VM. A Figura 5(b) apresenta o período de indisponibilidade de acesso à maquina virtual proporcional ao tempo total do trajeto do veículo. Percebe-se uma significativa melhora nos valores dessa métrica para todos os grupos de veículos avaliados com o aumento do tempo de predição. Para a média geral, percebe-se uma diminuição de até $40 \%$ no tempo de indisponibilidade total durante os trajetos dos veículos.

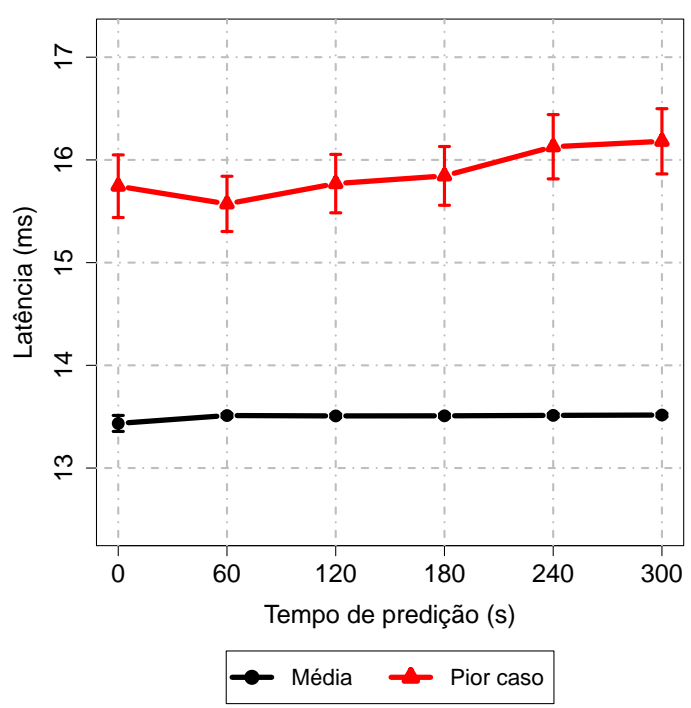

(a) Latência

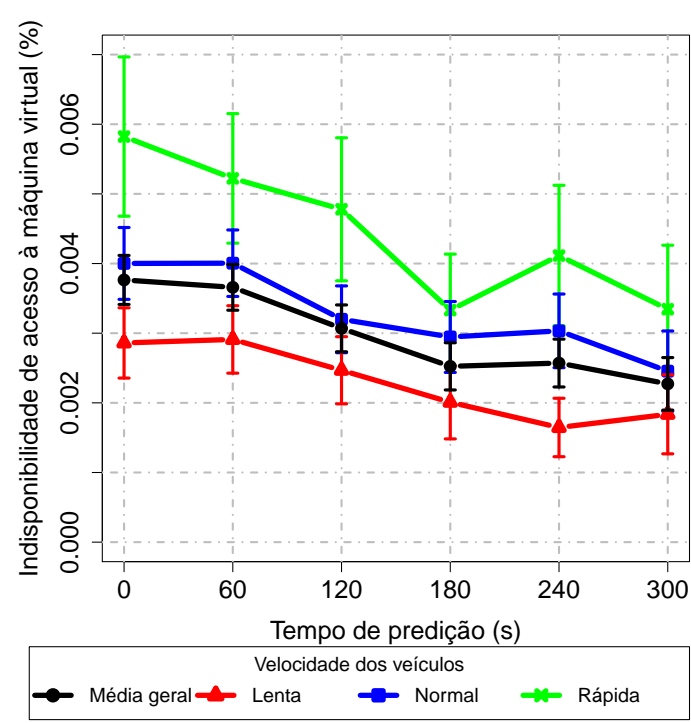

(b) Período proporcional de indisponibilidade de acesso à máquina virtual

Figura 5. Latência e tempo de indisponibilidade da aplicação

\section{Conclusão}

Gerenciamento e alocação de recursos da névoa tem se mostrado um dos grandes desafios a serem cumpridos a fim de oferecer uma qualidade de serviço aos usuários móveis conectados a essa infraestrutura. A migração de máquinas virtuais encontra-se nesse contexto. As atuais propostas pouco utilizam a inferência sobre a localização futura dos usuários durante esse processo de migração. Este trabalho contribui com uma análise sobre o processo de migração de máquinas virtuais em névoa tendo como base usuários móveis, como veículos.

Com base nas simulações desenvolvidas pode-se concluir que incorporar informações sobre o trajeto futuro do veículo pode reduzir o número de migrações necessárias para atender os clientes ao longo de seu trajeto sem comprometer a latência oferecida. Ter conhecimento sobre a localização aproximada do usuário nos próximos 5 minutos é suficiente para ajustar o destino da migração a ponto de diminuir em $20 \%$ o número total de migrações durante o todo o percurso do usuário. Com a redução no número de migrações, o tempo de indisponibilidade de acesso à máquina virtual causada durante cada migração, após a adoção de inferências sobre o trajeto futuro, pôde ser reduzida em até $40 \%$.

Espera-se desenvolver, dentre os trabalhos futuros, outras políticas de migração com base em cenários mais complexos, considerando a concorrência por recursos da 
névoa. Espera-se também realizar uma avaliação tendo como base dados para a inferência da localização futura dos usuários fornecidos por algoritmos reais de predição de mobilidade.

\section{Agradecimentos}

Este trabalho é parte do INCT sobre Internet do Futuro para Cidades Inteligentes (CNPq 465446/2014-0, CAPES 88887.136422/2017-00 e FAPESP 2014/50937-1).

Os autores gostariam de agradecer à CAPES pelo apoio financeiro e à Fundação de Amparo à Pesquisa do Estado de São Paulo (FAPESP), processo no ${ }^{\circ}$ 15/24494-8.

\section{Referências}

Ahmed, E., Gani, A., Sookhak, M., Ab Hamid, S. H., and Xia, F. (2015). Application optimization in mobile cloud computing: Motivation, taxonomies, and open challenges. Journal of Network and Computer Applications, 52:52-68.

Batista, D. M., Goldman, A., Hirata, R., Kon, F., Costa, F. M., and Endler, M. (2016). Interscity: Addressing future internet research challenges for smart cities. In Network of the Future (NOF), 2016 7th International Conference on the, pages 1-6. IEEE.

Bittencourt, L. F., Lopes, M. M., Petri, I., and Rana, O. F. (2015). Towards virtual machine migration in fog computing. In P2P, Parallel, Grid, Cloud and Internet Computing (3PGCIC), 2015 10th International Conference on, pages 1-8. IEEE.

Bonomi, F., Milito, R., Zhu, J., and Addepalli, S. (2012). Fog computing and its role in the internet of things. In Proceedings of the first edition of the MCC workshop on Mobile cloud computing, pages 13-16. ACM.

Codeca, L., Frank, R., and Engel, T. (2015). Luxembourg sumo traffic (lust) scenario: 24 hours of mobility for vehicular networking research. In Vehicular Networking Conference (VNC), 2015 IEEE, pages 1-8. IEEE.

Gomes, A. S., Braun, T., and Monteiro, E. (2016a). Enhanced caching strategies at the edge of lte mobile networks. In 2016 IFIP Networking Conference (IFIP Networking) and Workshops, pages 341-349. IEEE.

Gomes, A. S., Fonseca, V., Sousa, B., Palma, D., Simoes, P., Monteiro, E., and Cordeiro, L. (2016b). A mobile follow-me cloud content caching model. In NOMS 2016-2016 IEEE/IFIP Network Operations and Management Symposium, pages 763-766. IEEE.

Gupta, H., Vahid Dastjerdi, A., Ghosh, S. K., and Buyya, R. (2017). ifogsim: A toolkit for modeling and simulation of resource management techniques in the internet of things, edge and fog computing environments. Software: Practice and Experience, 47(9):1275-1296.

Júnior, W., Henrique, A., and Lopes, K. (2016). Avaliação de desempenho da técnica de offloading computacional em nuvens móveis. wperformance.

Kim, O. T. T., Tri, N. D., Nguyen, V., and Hong, C. S. (2015). Addressing virtual machine migration problems in cloud based vehicular networks.

Lopes, M. M., Higashino, W. A., Capretz, M. A., and Bittencourt, L. F. (2017). Myifogsim: A simulator for virtual machine migration in fog computing. In Companion Proceedings of the 10th International Conference on Utility and Cloud Computing, UCC ' 17 Companion, pages 47-52, New York, NY, USA. ACM. 
Malandrino, F., Casetti, C., Chiasserini, C.-F., and Fiore, M. (2014). Content download in vehicular networks in presence of noisy mobility prediction. IEEE Transactions on Mobile Computing, 13(5):1007-1021.

Mell, P. M. and Grance, T. (2011). SP 800-145. The NIST Definition of Cloud Computing. Technical report, Gaithersburg, MD, United States.

Mustafa, A. M., Abubakr, O. M., Ahmadien, O., Ahmedin, A., and Mokhtar, B. (2017). Mobility prediction for efficient resources management in vehicular cloud computing. In Mobile Cloud Computing, Services, and Engineering, 5th IEEE International Conference on, pages 53-59. IEEE.

Nabi, T., Mittal, P., Azimi, P., Dig, D., and Tilevich, E. (2015). Assessing the benefits of computational offloading in mobile-cloud applications. In Proceedings of the 3rd International Workshop on Mobile Development Lifecycle, pages 17-24. ACM.

Puliafito, C., Mingozzi, E., and Anastasi, G. (2017). Fog computing for the internet of mobile things: issues and challenges. In Smart Computing (SMARTCOMP), 2017 IEEE International Conference on, pages 1-6. IEEE.

Refaat, T. K., Kantarci, B., and Mouftah, H. T. (2014). Dynamic virtual machine migration in a vehicular cloud. In 2014 IEEE Symposium on Computers and Communications (ISCC), pages 1-6. IEEE.

Roman, R., Lopez, J., and Mambo, M. (2016). Mobile edge computing, fog et al.: A survey and analysis of security threats and challenges. Future Generation Computer Systems.

Satyanarayanan, M., Bahl, P., Caceres, R., and Davies, N. (2009). The case for vm-based cloudlets in mobile computing. IEEE pervasive Computing, 8(4):14-23.

Shin, W., Min, B.-Y., and Kim, D. K. (2015). Vehicaching: Embracing user request on vehicle route with proactive data transportation. In 2015 IEEE 81st Vehicular Technology Conference (VTC Spring), pages 1-5. IEEE.

Taleb, T. and Ksentini, A. (2013). Follow me cloud: interworking federated clouds and distributed mobile networks. IEEE Network, 27(5):12-19.

Yan, H., Wan, J., Wang, Y., Wang, Z., and Song, Z. (2013). Vehicular cyber-physical systems with mobile cloud computing support. In International Conference on Cloud Computing, pages 27-35. Springer.

Yao, H., Bai, C., Zeng, D., Liang, Q., and Fan, Y. (2015). Migrate or not? exploring virtual machine migration in roadside cloudlet-based vehicular cloud. Concurrency and Computation: Practice and Experience, 27(18):5780-5792.

Yu, R., Zhang, Y., Gjessing, S., Xia, W., and Yang, K. (2013a). Toward cloud-based vehicular networks with efficient resource management. IEEE Network, 27(5):48-55.

Yu, R., Zhang, Y., Wu, H., Chatzimisios, P., and Xie, S. (2013b). Virtual machine live migration for pervasive services in cloud-assisted vehicular networks. In Communications and Networking in China (CHINACOM), 2013 8th International ICST Conference on, pages 540-545. IEEE. 